\title{
I tempi della tiroidectomia nella MEN2
}

\author{
Alessandro Prete ${ }^{1} \cdot$ Rossella Elisei $^{1}$
}

Accettato: 26 marzo 2020 / Pubblicato online: 21 giugno 2021

(C) The Author(s) 2021

\section{Introduzione}

Il carcinoma midollare della tiroide (CMT) è un tumore raro e rappresenta circa il $5-10 \%$ di tutti i tumori della tiroide. Circa il $75 \%$ dei CMT sono sporadici mentre il $25 \%$ sono familiari, i quali sono causati in più del $99 \%$ dei casi da una mutazione germinale del gene REarranged during Transfection $(R E T)$. Le forme familiari di CMT vengono distinte in Multiple Endocrine Neoplasia (MEN) tipo 2A e 2B quando associate ad altre neoplasie endocrine. La MEN2A poi, in base all' associazione di alcune caratteristiche fenotipiche, viene distinta in: MEN2A classica, nella quale oltre al CMT può essere presente il feocromocitoma (FEO) e l'iperparatiroidismo da iperplasia e/o adenomatosi delle paratiroidi; MEN2A associata a lichen cutaneo amiloidosico e MEN2A con malattia di Hirschsprung. La MEN2B è invece caratterizzata da un fenotipo molto peculiare con habitus marfanoide, neurinomi mucosi, labbra carnose, megacolon e FEO. Infine, esiste una forma familiare di CMT isolato (FMTC) senza altre patologie associate [1]. Le sindromi MEN2 rappresentano una sfida nella gestione sia del CMT che delle patologie correlate. In particolare, in considerazione dell'elevata penetranza di sviluppo del CMT, la cui guarigione definitiva dipende dalla precocità della diagnosi e, quindi, della tiroidectomia, risulta fondamentale stabilire il tempo migliore per eseguire l'intervento chirurgico.

R. Elisei

rossella.elisei@med.unipi.it

1 Dipartimento di Medicina Clinica e Sperimentale, Università di Pisa, Divisione di Endocrinologia, Azienda Ospedaliero Universitaria Pisana, Pisa, Italia

\section{Il timing della tiroidectomia: profilattica o precoce?}

Con il concetto di tiroidectomia profilattica si intende la rimozione della tiroide prima che il CMT possa svilupparsi, mentre si parla di tiroidectomia precoce se eseguita quando il CMT è presente ma ancora confinato all'interno della tiroide e prima che possa manifestarsi clinicamente. In considerazione dell'elevata penetranza della patologia, si potrebbe concludere di eseguire l'intervento chirurgico nei pazienti portatori della mutazione germinale del gene RET non appena noto il loro stato mutazionale. Oggi lo stato di portatore della mutazione è identificabile già in età pediatrica o addirittura in epoca prenatale e, quindi, si potrebbe pensare a una tiroidectomia profilattica già in epoca neonatale o pediatrica. Tuttavia, bisogna considerare che i bambini sono maggiormente gravati da complicanze della tiroidectomia, quali ipoparatiroidismo e lesione del nervo laringeo ricorrente, e che a seguito della tiroidectomia i pazienti sono destinati a una terapia sostitutiva vita natural durante della funzione tiroidea $\mathrm{e}$, eventualmente, paratiroidea [2]. Pertanto, è necessario valutare il momento migliore per eseguire una tiroidectomia che sia precoce e curativa e non necessariamente profilattica.

\section{Fattori biologici}

Un elemento fondamentale da tenere in considerazione nella valutazione del timing della tiroidectomia è il tipo di mutazione di RET: non tutte le mutazioni hanno la stessa attività trasformante che si traduce, poi, in una diversa aggressività del CMT (Fig. 1) [3]. Oggi è noto che la mutazione tipica della MEN2B, e cioè la mutazione germinale M918T, è quella con la maggiore attività trasformante e per tale motivo, come è noto dalla clinica, i pazienti con MEN2B hanno generalmente un CMT molto aggressivo. Tra le mutazioni 


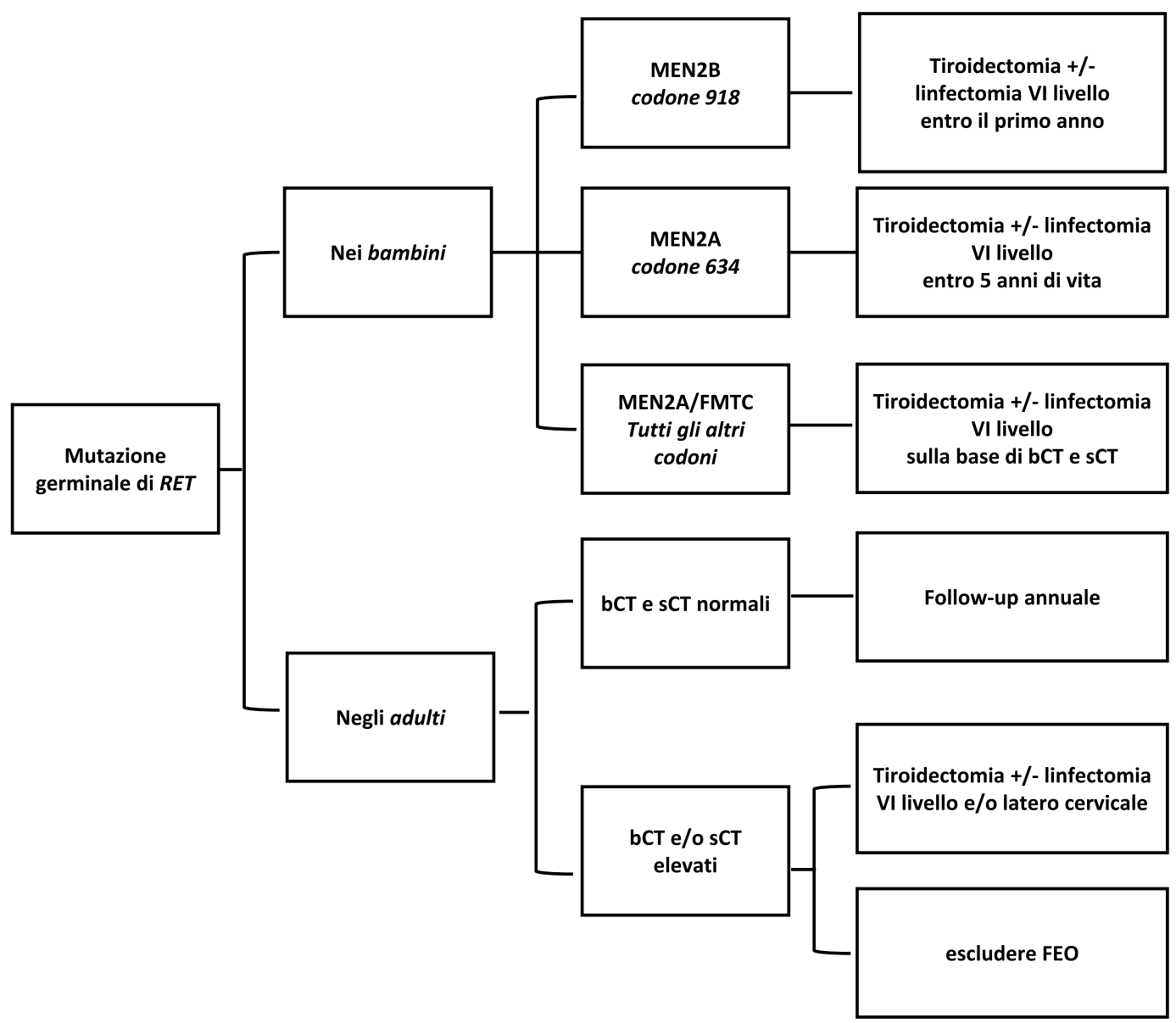

Fig. 1 Algoritmo di trattamento dei soggetti portatori di una mutazione germinale di RET

principalmente responsabili della MEN2A, le mutazioni al codone 634 sono le più aggressive e generalmente associata alla MEN2A classica. Tutte le altre mutazioni sono considerate a moderata aggressività e tra queste alcune, tipo le mutazioni al codone 790, sono tra le meno aggressive. Questo diverso comportamento biologico si traduce non solo in una diversa aggressività della malattia ma anche in una epoca di insorgenza diversa: sicuramente più precoce per le mutazioni più trasformanti e più tardiva per le altre.

Occorre poi considerare che il CMT produce la calcitonina $(\mathrm{CT})$, che è un marcatore specifico della patologia, e che c'è una stretta correlazione tra valore circolante di Ct ed estensione della malattia. In particolare, studi recenti mostrano che pazienti con CT basale preoperatoria al di sopra dei valori normali ma inferiore a $40 \mathrm{pg} / \mathrm{mL}$ hanno generalmente un CMT intratiroideo senza evidenza di metastasi al comparto centrale $[4,5]$. È quindi evidente che la misurazione dei livelli circolanti di CT entra fortemente in gioco nella decisione di intervenire chirurgicamente o meno, tenendo conto che per valori nei limiti della norma e comunque al di sotto di $40 \mathrm{pg} / \mathrm{mL}$, il CMT o non si è ancora sviluppato o, se lo è, è ancora intratiroideo e, quindi, suscettibile di guarigione clinica dopo tiroidectomia precoce.

\section{Fattori epidemiologici}

È abbastanza intuitivo che il fattore età è molto importante nella decisione terapeutica. Infatti, se da un lato è vero che la presenza della mutazione di $R E T$ ha un'elevatissima specificità e sensibilità per la diagnosi di CMT, è però anche vero che l'età di sviluppo del CMT, come precedentemente detto, è diversa in base al tipo di mutazione. La domanda, quindi, è se i portatori di una mutazione germinale di RET devono sempre e comunque essere sottoposti a tiroidectomia immediatamente dopo la loro identificazione. Come indicato dalle linee guida internazionali [6], certamente occorre procedere a tiroidectomia quanto prima, se possibile entro il primo anno di vita, nel caso di una MEN2B, valutando una linfoadenectomia del compartimento centrale dopo aver identificato e preservato le paratiroidi. Un recente studio internazionale, multicentrico e retrospettivo ha raccolto la storia naturale, il trattamento e il follow-up di 345 pazienti affetti da MEN2B [7]. Questo studio ha confermato che i bambini trattati con tiroidectomia prima del compimento del primo anno di vita hanno una probabilità di remissione clinica di malattia molto maggiore rispetto a quelli operati dopo. Tuttavia, solo il $16 \%$ dei casi è costituito da figli di soggetti affetti da MEN2B, 
e quindi individuabili alla nascita pe mezzo di screening. La gran parte dei pazienti MEN2B presentano mutazioni de novo con genitori perfettamente sani. A seguito di ciò, nonostante le caratteristiche fisiche peculiari dei bambini affetti da MEN2B, troppo spesso questi pazienti vengono diagnosticati tardivamente [8] quando il CMT è già avanzato. Nel caso di mutazioni al codone 634, che generalmente sottintendono una sindrome MEN2A classica, le linee guida internazionali suggeriscono l'intervento a 5 anni di età o prima, tenendo conto dei valori di $\mathrm{Ct}$ basale. In tutti gli altri casi sia di MEN2A che di FMTC, e quindi di tutte le mutazioni considerate a moderata aggressività, l'intervento può essere procrastinato all'evidenza di valori elevati di $\mathrm{Ct}$, discutendo questo approccio di sorveglianza attiva, che prevede controlli periodici della calcitonina basale e/o dopo stimolo, con il paziente se adulto o con i genitori se minore [6].

\section{Valutazione delle neoplasie endocrine associate al CMT}

All'interno della valutazione delle patologie correlate, meritano estrema attenzione il FEO e l'iperparatiroidismo. Le linee guida consigliano di intraprendere lo screening del FEO con metanefrine e normetanefrine urinarie dall'età di 11 anni nei pazienti con mutazione di RET a carico dei codoni 634 e 918, dall'età di 16 anni negli altri casi. Per quanto riguarda l'iperpartiroidismo, le linee guida raccomandano di iniziare lo screening all'età di 11 anni in caso di mutazione dei codoni 634 e 918, a 16 anni negli altri casi [6]. È inoltre da segnalare che ad oggi, escluso qualche raro caso di FEO in famiglie con mutazione V804M, nessun caso di associazione di FEO e/o iperparatiroidismo è stato segnalato nelle famiglie con mutazioni non-cisteiniche. È quindi da valutare se nel caso di tali mutazioni vi sia un costo-beneficio nel fare lo screening annuale di tali patologie associate.

\section{Conclusioni}

La gestione dei casi di MEN2 è estremamente complessa e deve comprendere sia la gestione del CMT, sia delle patologie associate alle sindromi. Mentre nei casi di MEN2A e dell'FMTC il timing dell'intervento chirurgico e la sua estensione sono dipendenti dal codone mutato del gene RET e dai valori di $\mathrm{Ct}$ basale e dopo stimolo, i pazienti affetti da MEN2B devono essere sottoposti a tiroidectomia profilattica entro il primo anno di vita o quanto prima se diagnosticati più tardivamente.

Funding Note Open access funding provided by Università di Pisa within the CRUI-CARE Agreement.
Conflitto di interesse Gli autori Alessandro Prete e Rossella Elisei dichiarano di non avere conflitti di interesse.

Consenso informato Lo studio presentato in questo articolo non ha richiesto sperimentazione umana.

Studi sugli animali Gli autori di questo articolo non hanno eseguito studi sugli animali.

Nota della casa editrice Springer Nature rimane neutrale in riguardo alle rivendicazioni giurisdizionali nelle mappe pubblicate e nelle affiliazioni istituzionali.

Open Access This article is licensed under a Creative Commons Attribution 4.0 International License, which permits use, sharing, adaptation, distribution and reproduction in any medium or format, as long as you give appropriate credit to the original author(s) and the source, provide a link to the Creative Commons licence, and indicate if changes were made. The images or other third party material in this article are included in the article's Creative Commons licence, unless indicated otherwise in a credit line to the material. If material is not included in the article's Creative Commons licence and your intended use is not permitted by statutory regulation or exceeds the permitted use, you will need to obtain permission directly from the copyright holder. To view a copy of this licence, visit http://creativecommons.org/licenses/by/4.0/.

\section{Bibliografia}

1. Romei C, Pardi E, Cetani F, Elisei R (2021) Genetic and clinical features of multiple endocrine neoplasia types 1 and 2. J Oncol 2012:705036

2. Sosa JA, Tuggle CT, Wang TS et al (2008) Clinical and economic outcomes of thyroid and parathyroid surgery in children. J Clin Endocrinol Metab 93:3058-3065

3. Cosci B, Vivaldi A, Romei C et al (2011) In silico and in vitro analysis of rare germline allelic variants of RET oncogene associated with medullary thyroid cancer. Endocr-Relat Cancer 18(5):603-612

4. Elisei R, Romei C, Renzini G et al (2012) The timing of total thyroidectomy in RET gene mutation carriers could be personalized and safely planned on the basis of serum calcitonin: 18 years experience at one single center. J Clin Endocrinol Metab 97:426-435

5. Rohmer V, Vidal-Trecan G, Bourdelot A et al (2011) Prognostic factors of disease-free survival after thyroidectomy in 170 young patients with a RET germline mutation: a multicenter study of the Groupe Français d'Etude des Tumeurs Endocrines. J Clin Endocrinol Metab 96(3):E509-E518

6. Wells SA, Asa SL, Dralle H et al (2015) Revised American Thyroid Association guidelines for the management of medullary thyroid carcinoma. Thyroid 25(6):567-610

7. Castinetti F, Waguespack SG, Machens A et al (2019) Natural history, treatment, and long-term follow up of patients with multiple endocrine neoplasia type 2B: an international, multicentre, retrospective study. Lancet Diabetes Endocrinol 7(3):213-220

8. Elisei R, Matrone A, Valerio L et al (2019) Fifty years after the first description, MEN 2B syndrome diagnosis is still late: descriptions of two recent cases. J Clin Endocrinol Metab 104(7):2520-2526 